\title{
ALGUNAS TENDENCIAS DEL DERECHO INTERNACIONAL A PRINCIPIOS DEL SIGLO XXI
}

\author{
Enrique LAGOS*
}

RESUMEN: La primera parte del artículo se refiere a las características de un mundo "uni-multipolar complejo" y a la discusión central acerca de la globalización. En la segunda parte se pone de relieve el impacto del mundo globalizado en el contenido y desarrollo del derecho internacional, resaltando algunas características relevantes en cuanto a los nuevos sujetos que comparten roles con los Estados; la ampliación y fragmentación del objeto de estudio del derecho internacional; las nuevas modalidades en el proceso de elaboración de las fuentes, así como la expansión de la figura del soft law; y los avances en materia de la responsabilidad internacional. Finalmente, en la última parte se presentan algunos alcances de los nuevos desarrollos del derecho internacional en el ámbito del sistema interamericano.

ABSTRACT: The first part of the article describes the "uni-multipolar complex" world and the central debate over globalization and its consequences, not only economic but political, social, and cultural as well. The second part examines the impact of globalization on the content and development of international law. It focuses on certain important characteristics of the new legal persons sharing roles with the States; the expansion and fragmentation of questions examined in international law; new approaches to generating sources of law and the broadening concept of "soft law"; and progress in the area of international liability. The last section gives some perspectives on new developments in international law within the inter-American system.

RÉSUMÉ: La première partie de l'article traite des caractéristiques d'un monde "uni-multipolaire complexe" et du débat central ouvert sur la mondialisation et ses conséquences. Dans la deuxième partie est mis en relief l'impact d'un monde globalisé sur le contenu et l'évolution du droit international, et souligne certaines caractéristiques pertinentes relevant des dossiers dominant l'actualité qui partagent les rôles avec les États; l'élargissement et la fragmentation de l'objet de l'étude du droit international; les nouvelles modalités du processus d'élaboration des sources, ainsi que l'expansion du concept du soft law, et les progrès accomplis en matière de responsabilité internationale. Enfin, dans la dernière partie sont envisagées certaines perspectives de nouveaux développements du droit international au sein du Système Interaméricain.

* Subsecretario de Asuntos Jurídicos de la Organización de Estados Americanos. 
SUMARIO: I. Introducción. II. Un nuevo entorno internacional y sus principales características. III. Impacto de la actual realidad mundial en el derecho internacional. IV. El derecho internacional en el marco del sistema interamericano. V. Conclusiones.

\section{INTRODUCCIÓN}

El siglo XXI comienza con grandes transformaciones en diversos ámbitos de la sociedad contemporánea. Cambios que se gestaron fundamentalmente a finales del siglo XX, cuyas consecuencias configuran un nuevo panorama para la comunidad internacional. El proceso globalizador, partícipe y, en la mayoría de los casos, constructor de esta nueva realidad mundial, ha planteado nuevos retos y reconfigurado estructuras, conceptos, modelos y valores, entre otros aspectos.

Uno de los ámbitos impactados es desde luego el jurídico, el cual experimenta transformaciones sustanciales, no sólo a nivel conceptual, sino práctico. Una de sus áreas, quizá de mayor cambio, es hoy en día el derecho internacional, cuya vigencia y desarrollo se han visto afectados no tan sólo por la globalización, sino también por el nuevo orden mundial.

En este sentido, este trabajo tiene como objetivo evidenciar y reflexionar sobre este nuevo panorama al cual se enfrenta la vigencia y desarrollo del derecho internacional. Así hemos dividido nuestro estudio en tres partes. La primera refiere las principales características de la realidad internacional de cara a la globalización. En la segunda parte, se pondrá de relieve como viene impactando el mundo globalizado en la estructura y desarrollo del derecho internacional, resaltando algunas características relevantes. Finalmente, en la tercera y última parte, se delinearán los alcances de las transformaciones existentes en el ámbito interamericano.

\section{EL NUEVO ENTORNO INTERNACIONAL Y SUS PRINCIPALES CARACTERÍSTICAS}

Como señalamos en la introducción, el inicio de un nuevo milenio se presenta bajo el signo de la globalización ${ }^{1}$ y el profundo cambio del or-

1 Existe numerosa bibliografía sobre el tema de la globalización, principalmente desde el punto de vista del análisis económico, aunque en realidad apreciamos que la globalización es 
den mundial, resultado de los ataques terroristas ocurridos el 11 de septiembre del 2001 y de las incursiones armadas a Afganistán e Irak; hechos que han impactado decididamente en la estructura de la comunidad internacional aún vigente.

En lo referente al proceso de la globalización, éste viene desplegando sus efectos no sólo a nivel internacional sino también en el ámbito interno de los Estados, permeando profundamente los distintos aspectos del quehacer humano y poniendo en cuestión las categorías y los conceptos tradicionales a todo nivel. Por lo tanto, una reflexión inicial obligada o de la mayor significación estará necesariamente referida a reseñar qué significa vivir en un mundo globalizado.

\section{Globalización y revolución tecnológica}

Una respuesta inmediata está dada por la constatación de que la globalización implica un mundo crecientemente interconectado en múltiples ámbitos, en gran medida, debido a la revolución tecnológica-informáti$\mathrm{ca}^{2}{ }^{2}$ ligada fundamentalmente a las telecomunicaciones y sistemas cibernéticos, en una suerte de "tercera revolución industrial" como algunos la denominan. Desde el establecimiento de los sistemas de intercambio electrónico en 1971, pasando por el lanzamiento del primer satélite directo de comunicaciones en 1976, el uso comercial de los cables de fibra óptica en 1977 y la introducción a nivel mundial de la red Web en 1991, la tecnología cibernética, en variadas y sofisticadas aplicaciones, ha seguido expandiéndose a lo largo y ancho del planeta. ${ }^{3}$ Todo ello ha permitido la creación de "comunidades virtuales" a través de las denominadas networks, ámbito en el cual no tienen significación las fronteras nacionales.

La globalización acompañada de la revolución informática, por un lado, ha ido transformando nuestra capacidad, no sólo para producir e in-

política, tecnológica y cultural, además de económica. Entre otros autores que analizan este fenómeno puede mencionarse a los siguientes: Touraine, Alain, Podremos vivir juntos? Iguales o diferentes, Buenos Aires, Fondo de Cultura Económica, 1996; Tomassini, Luciano, "El proceso de globalización y sus impactos socio-políticos", en Urzúa, Raúl (ed.), Cambio social y políticas públicas, Santiago de Chile, Universidad de Chile, Centro de Análisis de Políticas Públicas, 1997; Hoogvelt, Ankie, Globalization and the Post-Colonial World, Londres, Macmillan Press, 1997.

2 Nye, Joseph S., The Paradox of American Power, Oxford-Nueva York, Oxford University Press, 2002 (cáp. 2. The Information revolution).

3 Naisbitt, John, Megatrends. Global Paradox, Nueva York, Avon Books: "Digital technology is the technology that will reinvent the way people live, work and entertain themselves". 
tercambiar bienes y servicios, sino también para comunicarnos fácilmente y en forma casi instantánea con una gran diversidad de sujetos, en todas las latitudes; por el otro, nos ha brindando la posibilidad de tener acceso a toda clase de información. Otro aspecto que se debe resaltar es que éste acelerado crecimiento de las comunicaciones también ha sido posible por la enorme reducción de los costos de acceso y transmisión de la información. ${ }^{4}$

\section{Globalización, poder e interdependencia}

En cuanto a los efectos globalizadores sobre el nivel político de la sociedad internacional actual, debemos sin duda referirnos al surgimiento de nuevas modalidades de poder. Esto es, además del poder tradicional ejercido por los Estados, y el más contemporáneo de las organizaciones internacionales, existe ahora también un poder "difuso" (soft power como le denomina Joseph Nye $)^{5}$ que tiene características diferentes al poder tradicional y directo que se ejerce a nivel político y, sobre todo, en el ámbito del predominio militar. Este "poder difuso" refleja la interdependencia del mundo actual, y se manifiesta en forma extendida y horizontal, ejerciendo cada día su influencia en cientos de millones de individuos, a través de la propagación de determinados valores promovidos por los Estados, las organizaciones internacionales, y de manera creciente, por las organizaciones no-gubernamentales, empresas y diversas entidades de la sociedad civil de la mas distinta naturaleza, que han ido adquiriendo una mayor presencia en el contexto internacional.

Por medio de un dinámico intercambio comunicacional, actualmente se difunden valores, ideas, cultura, productos y todo tipo de servicios. El liberalismo político y económico —ideología predominante-, se viene expandiendo rápidamente a todo lo largo y ancho del planeta, precisamente a través de la Internet, del cine, de la televisión, del cable y el satélite, la música, la moda y la propaganda y venta de miles de productos representativos de la sociedad occidental, conformando una suerte de poder paralelo al que se ejerce formalmente a través de los medios tradicionales, sean políticos, institucionales o militares.

4 Nye, Joseph, op. cit., nota 2.

5 Ibidem, p. 11: "Soft power is more than just cultural power... soft power is likely to become increasingly important in the global information age of this new century". 
Este fenómeno tiene obviamente impactos positivos y negativos, puesto que se trata de relaciones trasnacionales que mayormente no pueden ser controladas por los Estados y gobiernos. Así, por un lado, tenemos el cotidiano y amplio acceso de millones de personas a la información, al conocimiento, a la cultura, a los servicios y productos de todo tipo, pero al mismo tiempo, se expanden a través de la Internet, las redes delictivas del narcotráfico, del terrorismo, del crimen organizado y de los delitos propiamente cibernéticos como la piratería informática y la pornografía infantil, aprovechando éstos avances tecnológicos de frontera.

Asimismo, no podemos olvidar que si bien cada día millones de personas en los lugares más remotos pueden beneficiarse de los avances de las sociedades desarrolladas; también hay muchas otras que se van quedando relegados en una suerte de "analfabetismo cibernético", con el peligro de que se amplíe rápidamente la brecha existente entre quienes tienen o no tienen aún acceso a la tecnología.

En definitiva, esta nueva era de "información globalizada" nos convierte cada día más en sujetos interdependientes, quedando notablemente disminuido el significado tradicional de las fronteras geográficas en una configuración que cada vez se parece más a lo que algunos han denominado como la "aldea global". ${ }^{6}$

\section{Globalización y economía abierta}

Otro ámbito esencial del actual panorama internacional es, sin duda alguna, el económico. Hablar de globalización es reafirmar el hecho de que somos interdependientes económicamente. Los cambios en este campo han sido de una magnitud sin paralelo en tres aspectos principales: el intercambio económico de bienes y servicios, o sea, la internacionalización del comercio; la globalización de las empresas multinacionales a través de adquisiciones y fusiones; y la globalización de los flujos de capital en el sistema financiero internacional. Como el Fondo Monetario Internacional ${ }^{7}$ define en un estudio, "la globalización es la rápida integra-

6 Ianni, Octavio, Teorías de la globalización, México, Siglo XXI Editores, 1999. Este autor sostiene que está en curso una homogeneización progresiva en las sociedades debido a los efectos de la globalización, y citando a Theodore Levitt agrega que: "en todos los lugares todo se parece cada vez más a todo, y más a medida que la estructura de preferencias del mundo es presionada hacia un punto común de homogeneización".

7 IMF, World Economic Outlook, mayo de 1997. 
ción de las economías a través del comercio, los flujos financieros, las difusión masiva de la tecnología de vanguardia, las networks informáticas, y los intercambios culturales".

En concordancia con esta realidad, la noción de una economía "nacional" se ha transformado, alterando la naturaleza misma de la producción y el comercio, y haciendo que en muchos casos el know-how sea más importante que el capital, la fuerza de trabajo y los recursos naturales. Asimismo, la universalización del comercio y la desregulación financiera han ido desarrollando un creciente mercado global. En la actualidad, tanto los gobiernos como las empresas se encuentran evaluando las ventajas de una economía mundial de libre mercado en un escenario de "regionalismo abierto", es decir un regionalismo no excluyente sino compatible con el seguimiento de acuerdos básicos de carácter mundial, como los propiciados en el marco de la Organización Mundial de Comercio $(\mathrm{OMC}){ }^{8}$

Sin embargo, se reconoce que los riesgos y desafíos de esta situación son también inmensos; y para muestra basta recordar las consecuencias negativas de la volatilidad de los capitales - por ejemplos, la crisis de Wall Street y la "crisis asiática", y el efecto "contagio" que puede trasmitirse a muchos mercados y países en pocas horas, desmantelando los sistemas financieros-. De igual forma, pueden mencionarse los desacuerdos e inequidades existentes en materia de libre comercio que se pusieron de manifiesto en la reciente Reunión de la Organización Mundial de Comercio (OMC), en Cancún, México (septiembre, 2003), ${ }^{9}$ no sólo a nivel de los países negociadores (Grupo G-22), sino también en las protestas protagonizadas por una heterogénea combinación de las ONG y grupos sociales contestatarios de la agenda de la OMC. ${ }^{10}$

8 Con relación a las negociaciones internacionales para compatibilizar la globalización mundial con los aspectos económicos, puede recordarse que la Ronda Uruguay (1986-1994) concluyó el 15 de abril de 1994 con la firma del acta final en la ciudad de Marrakech. El ciclo del GATT, de casi medio siglo, tendente a establecer progresivamente normas y códigos de conducta para un comercio más libre, leal y sin distorsiones, pasó a manos de la Organización Mundial del Comercio (OMC), que comienza sus actividades a partir del 1o. de enero de 1995, por medio de la cual se adquiere una institucionalización permanente de alcance universal para avanzar hacia el libre comercio a nivel global.

9 La OMC tuvo su ultima reunión en Cancún, México, a fines de 2003.

10 En un discurso del neozelandés Mike Moore, director general de la OMC, pronunciado ante las ONG en Seattle, en la Ronda del Milenio (1999), decía: "la OMC no es un gobierno mundial, ni un policía planetario ni un agente de los intereses empresariales; la OMC en una organización que actúa como mediadora en las diferencias comerciales. ¿Que es mejor? Dejar que la globalización 
A pesar de estos desafíos, apreciamos que la lógica del mercado es la que se impone y que, por tanto, la tendencia económica predominante sigue teniendo el signo de la apertura de los mercados y marcha aceleradamente hacia la globalización financiera y comercial.

\section{Globalización y multilateralismo}

Paralelamente al fenómeno antes mencionado, se ha venido conformando, particularmente después del fin de la guerra fría, un renovado auge del multilateralismo, con la realización de numerosas conferencias internacionales especializadas en diversos tipos de asuntos (género, pobreza, comercio, niñez, energía, etcétera), reuniones de grupos de países con intereses comunes (Grupo de Río, Cumbres de las Américas, OPEC, etcétera) y un expandido rol de las organizaciones internacionales con competencia para abordar cuestiones que afectan, en mayor o menor medida, a todas las naciones por igual. Existe la mayoritaria percepción de que determinados asuntos constituyen desafíos para la comunidad internacional en su conjunto, de manera tal que no pueden ser resueltos unilateralmente o exclusivamente con acciones a nivel interno, sino que necesitan de un enfoque común y coordinado, que sólo puede alcanzarse a través de la negociación y la voluntad política común de los gobiernos expresada a nivel multilateral. ${ }^{11}$

\section{Globalización y seguridad colectiva}

Pasando ahora al área de la seguridad colectiva, a diferencia de lo que sucede en el ámbito político y en el económico, en la actualidad puede apreciarse la aplicación de medidas unilaterales, superpuestas a las medidas de las organizaciones de seguridad colectiva existentes como la OTAN y los "Cascos Azules" de Naciones Unidas. Las acciones bélicas llevadas adelante por los Estados Unidos de América en Afganistán

proceda sin trabas, dominada por los más fuertes y regida por la "ley de la selva", o someterla a control mediante un sistema convenido de normas internacionales ratificadas por los gobiernos soberanos? El comercio no es la panacea para todos nuestros problemas, pero es parte de su solución. Luchamos por un sistema multilateral de comercio que sea un elemento esencial de la estructura de cooperación internacional. El mundo no sería un lugar más seguro sin las Naciones Unidas, el Banco Mundial, el FMI o la OMC". El Comercio Internacional en las Primeras Décadas del Siglo XXI, Seattle, OMC, 29 de noviembre de 1999.

11 Keohane, Robert y Nye, Joseph S., Poder e interdependencia, Buenos Aires, Grupo Editor Latinoamericano. 
$(2001-2002)^{12}$ y en Irak (2003), ${ }^{13}$ después de los condenables ataques terroristas del 11 de septiembre de 2001, demuestran el poder militar de una potencia y la puesta en práctica de un nuevo concepto del uso de la fuerza, el de "guerra preventiva", que se lleva adelante al tener como causal - ya no una amenaza cierta e inmediata - sino una más amplia, como es la que representa el terrorismo.

En este plano, cabe recordar que los países miembros de las Naciones Unidas estuvieron divididos en sus posiciones frente al rechazo de los Estados Unidos de América al sistema de inspecciones y verificaciones de armas de destrucción masiva llevado a cabo por los inspectores de la ONU, y su demanda de una intervención armada preventiva en Irak, debido a la supuesta existencia de armas de destrucción masiva. No obstante la oposición y las invocaciones de varios países desarrollados (Francia, Alemania, entre otros) así como la falta de apoyo en el Consejo de Seguridad, prevaleció el uso unilateral de la fuerza.

Estos hechos evidencian que en determinadas circunstancias, el marco multilateral, en este caso el de las Naciones Unidas, puede quedar inoperativo para garantizar la seguridad colectiva a través de medios como la disuasión, del diálogo y del consenso. Esta nueva dimensión también ha tenido consecuencias a nivel de otras entidades internacionales como la Comunidad Europea y la OTAN, cuyos miembros estuvieron divididos en sus posiciones frente al asunto de Irak. Sin embargo, posteriormente, en octubre de 2003, los Estados Unidos de América regresaron a negociar al foro multilateral, obteniendo una resolución aprobada por unanimidad $^{14}$ en el seno del Consejo de Seguridad de las Naciones Unidas, a favor de la reconstrucción y del apoyo a la formación de un gobierno propio e independiente para Irak, avalando implícitamente la ocupación temporal de las fuerzas armadas lideradas por ese país así como la presencia de tropas de otros países. ${ }^{15}$

En este sentido, puede rescatarse que en la actualidad, por la interdependencia existente, el "aval" de los organismos internacionales resulta

12 Véase, en página $W e b$ de la ONU (www.un.org), las resoluciones relativas a la incursión armada en Afganistán.

13 Véase todo lo relativo a las últimas resoluciones con relación a Irak en página Web de la ONU.

14 Kissinger, Henry A., "Needed: A sense of Common Purpose", The Washington Post, 2 de noviembre de 2003.

15 Véase resolución de la ONU que legitimó la presencia de los Estados Unidos de América en Irak. 
— tarde o temprano - indispensable para legitimar cualquier acción internacional en el área del mantenimiento de la paz y la seguridad internacionales.

\section{Un mundo "uni-multipolar complejo"}

Finalmente, por lo que respecta a esta primera parte, debemos mencionar que para denominar de algún modo a la actual estructura internacional, podríamos coincidir con aquellos autores que hablan de la existencia de un mundo "uni-multipolar complejo"16 o compuesto, es decir, en el marco de esta nueva era de globalización y revolución tecnológica, encontramos superpuestas a la vez, variadas modalidades de relacionamiento y distintos planos de ejercicio del poder político, económico y militar. Por un lado, se aprecia una unipolaridad militar; por otro lado, una multipolaridad económica; $\mathrm{y}$, además, una creciente diversidad y complejidad en el ejercicio del poder político, ya no solamente centralizado en los Estados, sino compartido por las organizaciones internacionales, grupos regionales (Comunidad Europea), entidades no-gubernamentales, empresas multinacionales e individuos. Situación que trae consigo cambios importantes, tanto en la estructura como en la agenda de la sociedad internacional, con impactos de diverso tipo, a diversos ámbitos y a todo nivel.

\section{IMPACTO DE LA ACTUAL REALIDAD MUNDIAL EN EL DERECHO INTERNACIONAL}

Las situaciones descritas nos ofrecen un escenario interesante para examinar cuáles son las consecuencias de las mismas, en el ámbito jurídico, cuyo desarrollo avanza al compás de los cambios que afectan a la sociedad mundial. Es decir, existe una compleja relación entre la formación del derecho internacional y la política, porque si bien, por un lado, el derecho se formula en directa relación con el poder político vigente en

16 Nye, Joseph, op. cit., nota 2, menciona el concepto para calificar al mundo actual como: "Uni-multipolar hibrid"; véase también Smith, Peter H., Talons of the Eagle, Nueva York-Oxford, Oxford University Press, 2000 (Introduction): "In the Cold War Era, the world was bipolar and the goal was geopolitical predominance through cultivation of ideological affinity; and today, in the Post-Cold-War Era, we perceive a combination of unipolar and multipolar powers with the goal to develop economic influence, security, cooperation and alignment". 
un determinado periodo histórico, por otro, el propio derecho influye en las relaciones de poder en la sociedad.

En el caso del derecho internacional actual, se perciben cambios a nivel de su objeto de estudio, de los sujetos, de las fuentes y de la responsabilidad internacional. Cabe señalar que el análisis de los efectos jurídicos de la globalización y de la realidad internacional en estos momentos, es un tema que recién está comenzando a ocupar la atención de los especialistas. ${ }^{17}$

Para hacer una apreciación de la evolución de nuestra disciplina y las principales características que presenta en esta primera fase del siglo XXI, tendremos como marco de referencia comparativo al derecho internacional que emerge después de la Segunda Guerra Mundial, moldeado según las características del poder y de la sociedad internacional de esa época; esto es, un derecho internacional centrado en las relaciones inter-estatales, basado en la coexistencia pacífica y marcado por el pluralismo ideológico. ${ }^{18}$

Recordemos que el mantenimiento de la paz y la seguridad internacionales entre Estados con distintos regímenes políticos, a través de una coexistencia pacífica, fue el objetivo principal que amparó la creación de las Naciones Unidas. La estructura de la organización mundial refleja el balance de poder existente en aquel entonces, a través, por ejemplo, del derecho de veto para sólo algunas naciones que tienen la categoría de miembros permanentes en el Consejo de Seguridad de la ONU (Estados Unidos de América, China, Francia, Rusia y Gran Bretaña). ${ }^{19}$ Posteriormente, después de la caída del muro de Berlín y el fin de la guerra fría, ha venido una "ola" de creciente democratización unida a la liberalización de las economías de la mayor parte de los Estados, que ya empieza a presionar un cambio jurídico a todo nivel, inclusive en lo que respecta a la actual estructura y sistema de decisiones de las Naciones Unidas. ${ }^{20}$

17 Fox, Gregory H. y Roth, Brad R. (eds), Democratic Governance and International Law, Cambridge University Press, 2000; Byers, Michael (ed.). The Role of Law in International Politics, Oxford University Press, 2000.

18 Véase Carrillo Salcedo, Juan Antonio, El derecho internacional en perspectiva histórica, Madrid, Tecnos, 1991.

19 Véase Carta de las Naciones Unidas, artículo 23.

20 Véase en página $\mathrm{Web}$ de la ONU (www.un.org) trabajos llevados adelante para reformar la estructura y sistema de toma de decisiones en las Naciones Unidas. 


\section{Nuevos sujetos del derecho internacional}

Veamos ahora lo relativo a los sujetos del derecho internacional. Como decíamos al comienzo, aunque los Estados sigan siendo los sujetos por excelencia del derecho internacional, ${ }^{21}$ hoy en día comparten su primacía con otros actores que han alcanzado una posición destacada en determinadas áreas y están adquiriendo una influencia cada vez mayor en el curso de los acontecimientos a nivel mundial.

Los Estados, con sus elementos constitutivos tradicionales de territorio, población, gobierno, soberanía e independencia, ${ }^{22}$ siguen siendo en gran medida los generadores de las dos principales fuentes del derecho internacional: la costumbre, que es la práctica generalizada aceptada como derecho, y los tratados. ${ }^{23}$ En este sentido, la mayor parte de las normas vigentes están principalmente dirigidas a regular las obligaciones de los Estados. Sin embargo, el derecho internacional tradicional creado por y para los Estados, tiene hoy que competir, como resultado de la globalización, con otros actores no-estatales que intervienen tanto en el proceso de elaboración de las normas como también en el plano de ser los destinatarios de dichas normas.

Las organizaciones internacionales, las comunidades supra-nacionales regionales (como la Comunidad Europea), las empresas multinacionales, las organizaciones no-gubernamentales, y el propio individuo, desempeñan en la actualidad distintos roles que influyen decididamente en el ámbito jurídico internacional.

Las organizaciones internacionales, en particular las Naciones Unidas, como sujetos del derecho internacional contemporáneo, representan un espacio tanto de legalidad como de legitimidad para avanzar hacia una comunidad mundial más solidaria que intente resolver conjuntamente los problemas que afectan a la humanidad. Muchos estiman que, teniendo en cuenta que vivimos en una sociedad multicultural, los foros multilaterales ofrecen la ventaja de que los Estados pueden agruparse en "bloques" en función de intereses comunes para negociar y alcanzar de-

21 Barberis, Julio A., Los sujetos del derecho internacional, Buenos Aires, Tecnos, 1984.

22 Llanos Mantilla, Hugo, Teoría y práctica del derecho internacional público, t. II: Derechos $y$ deberes fundamentales de los estados, Santiago de Chile, Editorial Jurídica de Chile, 1983.

23 El artículo 38 del Estatuto de la Corte Internacional de Justicia establece las fuentes del derecho internacional, mencionando, entre otras, las siguientes: a) Las convenciones internacionales, sean generales o particulares, que establecen reglas expresamente reconocidas por los Estados litigantes; b) La costumbre como una prueba de una práctica generalmente aceptada como derecho". 
terminados acuerdos, permitiendo asimismo una mayor publicidad y transparencia en los debates y negociación de nuevas normas. Así también, el marco multilateral brinda la posibilidad de un seguimiento más "ejecutivo" de los acuerdos alcanzados en una suerte de "monitoreo" internacional. Muchos tratados e incluso declaraciones y resoluciones, llevan aparejado mecanismos de seguimiento para evaluar y fortalecer el cumplimiento de dichos acuerdos.

Por otro lado, cabe anotar que el trabajo realizado en el marco de los organismos y de las conferencias internacionales ha ido generando la expansión del denominado "derecho informal" (soft law); es decir, el derecho que generalmente se expresa a través de declaraciones y resoluciones $\mathrm{y}$ acuerdos ejecutivos, abarcando una vastedad de temas y, dentro de este contexto, haciendo posible un marco de referencia temporal, necesario en una sociedad en permanente cambio, frente a la ausencia de normas consolidadas en tratados o través de la costumbre. ${ }^{24}$

En relación al individuo, en la actualidad éste no sólo es hoy beneficiario de la normatividad internacional, sino que puede, frente a la violación de sus derechos, reclamar ante una instancia supranacional competente, adquiriendo una subjetividad internacional "activa". ${ }^{25}$ Los instrumentos internacionales de derechos humanos brindan al individuo esta posibilidad en directa interrelación con distintas entidades supranacionales - comisiones, comités y tribunales de derechos humanos - que velan por la vigencia y protección de los derechos y libertades fundamentales. Por otro lado, también el individuo ha adquirido una subjetividad internacional "pasiva", cuando un tribunal internacional juzga directamente a determinadas personas por delitos o crímenes internacionales. Allí están los tribunales de Nuremberg y Tokio, conformados después de la

24 Con relación a las organizaciones internacionales, cabe anotar que en 1986 se aprobó la Convención de Viena sobre el Derecho de los Tratados entre Estados y Organizaciones Internacionales o entre Organizaciones Internacionales, desarrollo que está en consonancia con la actual realidad internacional.

25 Véase Pastor Ruidrejo, José, Curso de derecho internacional público, Madrid, Tecnos, 1986. 
Segunda Guerra Mundial; y los más contemporáneos como el Tribunal Internacional para la ex Yugoslavia ${ }^{26}$ y para Rwanda. ${ }^{27}$

En 1998, se produce otro nuevo e importante desarrollo con la aprobación del Estatuto para el establecimiento de una Corte Penal Internacional, ${ }^{28}$ indicativo de un desarrollo progresivo hacia la consolidación de una jurisdicción penal internacional.

En este recuento de los nuevos sujetos y normas vinculadas a ellos que surgen en el mundo contemporáneo, no puede dejar de mencionarse a las organizaciones no-gubernamentales y diversas instituciones y entidades no-estatales que juegan un papel cada día mas dinámico y significativo en el escenario mundial, sea como protagonistas o en una forma más indirecta, influenciando el curso de los acontecimientos y las decisiones que se adoptan a nivel internacional. ${ }^{29}$ Por ejemplo, allí están Transparency Internacional, Green Peace, Amnesty International, Human Rights Watch, The International Red Cross, para mencionar sólo algunas de ellas. Recordemos que la acción coordinada e influencia de estos grupos se ha ampliado notablemente a través de las redes de contacto, base de datos informáticas e intensas campañas dirigidas a la opinión pública para avanzar en sus objetivos y propósitos. La acción de las ONG y otras entidades han logrado que su participación esté hoy institucionalizada a nivel de las organizaciones internacionales donde tienen asegurado un espacio de participación en el curso de las negociaciones de los temas prioritarios de la agenda internacional.

26 El Tribunal Internacional para el ex Yugoslavia fue establecido en virtud de las resoluciones del Consejo de Seguridad de las Naciones Unidas: 808, de 22 de febrero de 1993, y 827, de 25 de mayo de 1993.

27 El Tribunal Internacional para Rwanda se creó por Resolución 955 del Consejo de Seguridad de las Naciones Unidas del 8 de noviembre de 1994. Cabe anotar que desde el punto de vista orgánico, tanto el tribunal para el ex Yugoslavia como para Rwanda son organismos subsidiarios del Consejo de Seguridad, según el artículo 29 de la Carta de la ONU. Sin embargo, los estatutos de estos tribunales consagran la independencia de los mismos en su funcionamiento con relación al Consejo de Seguridad. La única obligación que tienen los presidentes de dichos tribunales es la presentación de un Informe Anual ante el Consejo de Seguridad y ante la Asamblea General de la ONU.

28 En julio de 1998, tuvo lugar en Roma la Conferencia Diplomática de Plenipotenciarios de las Naciones Unidas sobre el "Establecimiento de una Corte Penal Internacional", que adoptó su estatuto que se compone de 128 artículos. Debe destacarse que la jurisdicción de esta Corte Penal Internacional tendrá un carácter complementario de las jurisdicciones penales nacionales.

29 Véase Krut, R., Globalization and Civil Society: NGO Influence in International Decision-Making, UN Research Institute for Social Development, 1997. 
Otros sujetos que no puede dejar de mencionarse son las empresas, en particular las multinacionales, que han pasado ha jugar un rol importante a nivel de la formación del derecho internacional, siendo algunas veces invitadas a las negociaciones en organismos y conferencias internacionales, para expresar su punto de vista en relación a determinados temas como, por ejemplo, los de carácter ambiental, los relativos a la energía, al transporte internacional, comercio y finanzas, lucha contra la corrupción, lavado de dinero, condiciones de trabajo y responsabilidad social de las empresas, entre otros. Se considera que el punto de vista empresarial debe ser tenido en cuenta para la elaboración de estándares internacionales y normas de conducta en una sociedad de mercado globalizada donde dichas compañías son protagonistas principales. ${ }^{30}$

\section{Ampliación del objeto de estudio del derecho internacional}

Un tema significativo en esta evolución que venimos reseñando, es que el objeto de estudio del derecho internacional se ha expandido de manera notable. Prácticamente no hay tema de preocupación a nivel interno que no se haya "internacionalizado" de uno u otro modo. La complejidad multicultural de las distintas sociedades compite paralelamente con las "fuerzas de homogeneización" generadas por la globalización. Todo ello ha llevado a una expansión normativa que abarca prácticamente todos los aspectos de la vida social: la paz y la seguridad internacional (conflictos armados y no armados) e interna ("seguridad ciudadana"); la solución pacífica de las controversias internacionales y el arreglo de todo tipo de conflictos; el derecho humanitario (aplicado a las guerras y también a los conflictos armados internos); la lucha contra la amenaza del terrorismo, del narcotráfico y de la corrupción; la contaminación y la protección ambiental; el régimen del espacio exterior; el derecho del mar; la institucionalidad democrática, incluyendo el monitoreo de los procesos electorales; los derechos humanos, la equidad de género y la no discriminación; la regulación del comercio y las finanzas; la distribución de la riqueza y la lucha contra la pobreza, entre otros, son todos temas prioritarios en la actual agenda internacional.

30 Véase Hossain, Kamal (ed.), Legal Aspects of the New International Economic Order, Londres, Pinter, 1980; Schachter, Oscar, "The decline of the Nation-State and its implications for International Law", Columbia Journal of Trasnational Law, 36, 1997. 
Precisamente éste fenómeno ha traído consigo una especie de "fragmentación" del derecho internacional, como producto de una compleja y variada gama de regímenes jurídicos y tribunales internacionales para atender a cada uno de los grandes temas objeto de regulación y reclamación internacional. Por el interés demostrado por los Estados desde 2001, esta temática ha sido incluida en el Programa de Trabajo de la Comisión de Derecho Internacional de las Naciones Unidas, bajo el título de "Fragmentación del derecho internacional: dificultades derivadas de la diversificación y expansión del derecho internacional". ${ }^{31}$

Dos áreas de estudio se derivan de la consideración de este asunto: Por un lado, los aspectos institucionales, es decir, todo aquello vinculado a la coordinación práctica, la jerarquía institucional y la necesidad de que los distintos tribunales judiciales y arbitrales internacionales ${ }^{32}$ tengan en cuenta sus respectivas jurisprudencias. Por otro lado, los aspectos sustantivos, relativos al examen de cómo es que el derecho se ha venido fragmentando en regímenes particulares (vale decir, en regímenes especiales (lex specialis), regímenes autónomos (self-contained regimes) y regímenes regionales) y la relación de los mismos con el derecho internacional general. La CDI se ha propuesto examinar en los próximos años sólo los aspectos sustantivos con la finalidad de elaborar recomendaciones para coadyuvar a la coherencia entre las normas del derecho internacional general y la de los regímenes particulares, tanto en los aspectos vinculados a la interpretación como a la aplicación de los mismos. ${ }^{33}$

\section{Las fuentes del derecho internacional}

En relación a las fuentes del derecho internacional, un primer aspecto que puede mencionarse es el relativo a las transformaciones que están dándose a nivel del proceso de elaboración de las normas, como consecuencia del impacto de la globalización, la notable ampliación de la agenda internacional y la acción de nuevos sujetos a la par de los Esta-

31 Véase Informe de la Comisión de Derecho Internacional, Naciones Unidas, 55 Periodo de Sesiones, mayo-junio y julio-agosto de 2003, cap. X: "Fragmentación del derecho internacional: dificultades derivadas de la diversificación y expansión del derecho internacional".

32 Véase idem. La CDI denomina a los tribunales judiciales y arbitrales (entre otros, Corte Internacional de Justicia de la Haya, Tribunal Internacional del Mar, Tribunal Europeo de Derechos Humanos, Corte Interamericana de Derechos Humanos, etcétera) como "operadores jurídicos".

33 Véase idem. El Grupo de Estudio de la CDI ya ha adoptado un calendario provisional para tratar el tema de la fragmentación de derecho internacional entre 2004 y 2006. 
dos. Una primera característica es la relativización del papel de la voluntad estatal en la formación del derecho internacional con un nuevo reparto de roles y responsabilidades internacionales. Como afirman algunos juristas, la interacción de distintos intereses expresados por diversos sujetos (Estados, organismos internacionales, ONG, grupos regionales, etcétera) está llevando a que "mientras una norma de la costumbre puede estar emergiendo, simultáneamente se la está codificando y desarrollando progresivamente en los trabajos de una conferencia internacional, que a su vez recoge la opinión surgida de las resoluciones de organismos internacionales y otros actos". ${ }^{34}$

En la actualidad, se ha acelerado la constatación de la práctica internacional y la consolidación de la opinio juris, muchas veces a través de la actividad normativa de las organizaciones internacionales y su cuasi proceso legislativo. Asimismo han adquirido gran relevancia las declaraciones, resoluciones, planes de acción, leyes y códigos modelo, acordadas en el ámbito multilateral, así como la jurisprudencia de diferentes tribunales internacionales.

En el ámbito de los principios que rigen el ordenamiento internacional, un primer nivel de análisis nos muestra que en la actualidad éstos parecen ir evolucionando hacia una concepción crecientemente "solidaria" del orden mundial, siguiendo una línea más dogmática, apegada a valores dominantes compartidos, y poniendo un mayor énfasis en la cooperación internacional como elemento fundamental, en lugar de una simple y llana "coexistencia pacifica". Esta concepción se ha ido gestando con el impacto de la interdependencia, la globalización y el multilateralismo, desarrollándose nuevos imperativos éticos, como por ejemplo el respecto irrestricto a los derechos humanos, entre ellos la libertad de expresión, la no-discriminación, la igualdad de géneros; el desarrollo sostenible; la solución pacífica de las controversias; el derecho de sufragio, es decir, la libertad de elegir y ser elegido para gobernar, entre otros.

En suma, la consolidación de estos nuevos principios está vinculada al interés común de salvaguardar valores compartidos tales como la paz y la seguridad internacionales, la libertad y la justicia, frente a nuevas amenazas como las del terrorismo; el narcotráfico; las armas de destrucción masiva; la contaminación ambiental; la depredación de las especies nati- 
vas; y la violación masiva de los derechos humanos. Naturalmente para esto se requieren consensos básicos y la adopción de acciones conjuntas en el marco de la solidaridad y de la cooperación internacional.

Una mención especial merece la expansión y el renovado auge de los valores democráticos y el rechazo de las formas autoritarias de gobierno. Las elecciones, como sabemos, que son sólo el primer paso en el camino hacia una sociedad democrática, se han realizado en los últimos diez años, en más de 180 países, trayendo a sus ciudadanos una mayor confianza y la esperanza de construir una cultura democrática donde los asuntos públicos y las políticas de desarrollo nacional sean conducidos en forma más responsable y transparente, respondiendo a los intereses prioritarios de toda la ciudadanía. Esta consolidación de un paradigma democrático a nivel de las fuentes del derecho internacional, puede apreciarse tanto a nivel universal como a nivel regional.

En el caso de las Naciones Unidas, cabe recordar que su carta constitutiva no contiene ninguna mención a la democracia, debido a que tenía que ser un instrumento jurídico que fuera consistente con la diversidad de regímenes políticos existentes en la época de su creación. Después de la aprobación de los dos pactos internacionales de derechos humanos, el de Derechos Civiles y Políticos y el de Derechos Económicos, Sociales y Culturales en 1966, es que comienzan a darse ciertos pronunciamientos y desarrollos teóricos conteniendo una mención a los principios democráticos, aunque siempre en conexión con el tema del respeto a los derechos humanos. Es sólo más recientemente, en el 2000, que se incluye expresamente en la Declaración del Milenio de las Naciones Unidas a valores como la libertad, la democracia y el gobierno participativo basado en la voluntad popular para asegurar los derechos humanos, como valores fundamentales para las relaciones internacionales en el siglo XXI. ${ }^{35}$

También es necesario señalar que la difusión y creciente consolidación de una visión liberal de los valores democráticos y de los derechos humanos ha afectado, a su vez, a otros principios tradicionales que antes eran "intocables". Uno de ellos es el relativo a la "soberanía del Estado",

35 United Nations Millennium Declaration, adopted by the General Assembly (A/55L.2), 8 de septiembre de 2000: "We consider certain fundamental values to be essential to international relations in the twenty-first century. These include: Freedom. Men and women have the right to live their lives and raise their children in dignity, free from hunger and from fear of violence, oppression and injustice. Democratic and participatory governance based on the will of the people best assures these rights". 
que se ha relativizado con la globalización y las nuevas características de la sociedad internacional. Por ejemplo, cuáles son hoy en día los asuntos que pueden ser considerados de "exclusiva competencia de la jurisdicción interna"? Hemos mencionado anteriormente que casi todos los temas de las agendas domésticas son considerados hoy en el ámbito multilateral, siendo objeto de una u otra forma de regulación internacional. Asimismo el nuevo "paradigma democrático" ha cambiado las nociones de legitimidad para el reconocimiento de gobiernos y su participación en el ámbito supra-nacional. Allí están las llamadas "cláusulas democráticas" en muchos acuerdos regionales que implican una nueva opinio iuris sobre el respeto al Estado de derecho y la gobernabilidad democrática. ${ }^{36}$

Por cierto que el principio de no-intervención, también se ha restringido significativamente. Así, la protección de los derechos humanos, y en algunos esquemas regionales, las formas democráticas de gobierno (Comunidad Europea y sistema interamericano), no permiten que los Estados invoquen en estas materias la excepción de asuntos de la jurisdicción interna (en el sentido del artículo 2, inciso 7 de la Carta de Naciones Unidas). ${ }^{37}$ Vale decir, para defender ciertos principios considerados en la actualidad como principios de jus cogens, ${ }^{38}$ que se acepta la implementación de una acción colectiva llevada adelante de acuerdo a las decisiones adoptadas por los Estados a nivel multilateral. La acción colectiva implica la actuación de un órgano internacional que, mediando ciertas circunstancias, puede avanzar una serie de medidas para apoyar la defensa de determinados valores. Así, puede utilizarse respecto de un país acosado por la ingobernabilidad o situaciones de crisis de la institu-

36 Véase las denominadas "cláusulas democráticas", por ejemplo en la Comunidad Europea; en las Cumbres de las Américas; Mercosur, etcétera. En las cuales se establece la exigencia de que los gobiernos que participan en estos acuerdos sean sólo aquellos elegidos democráticamente.

37 Carta de las Naciones Unidas, artículo 2, inciso 7: "Ninguna disposición de esta Carta autorizará a las Naciones Unidas a intervenir en los asuntos que son esencialmente de la jurisdicción interna de los estados, ni obligará a los Miembros a someter dichos asuntos a procedimientos de arreglo conforme a esta Carta; pero este principio no se opone a la aplicación de las medidas coercitivas prescritas en el Cap. VII".

38 La especificidad de los principios de jus cogens es que constituyen normas imperativas, absolutas y oponibles erga omnes (frente a todos) puesto que protegen los bienes fundamentales de la sociedad humana en un determinado periodo histórico. Las normas de jus cogens no admiten acuerdo en contrario, pudiendo ser sólo modificadas por una norma ulterior de derecho internacional general que tenga el mismo carácter. También puede decirse que son inderogables en tiempos de paz y en tiempos de guerra, y su violación configura crímenes internacionales. Véase artículo 53 de la Convención de Viena sobre el Derecho de los Tratados de 1969 y el Informe de la Comisión de Derecho Internacional en su 46o. Periodo de Sesiones (1994). 
cionalidad democrática, violaciones de los derechos humanos de la población, entre otros, existiendo el presupuesto de que ciertas situaciones graves necesitan, para ser resueltas de la mejor manera, el apoyo coordinado de la comunidad internacional organizada.

\section{Desarrollos a nivel de la responsabilidad internacional}

Otro ámbito en el cual el desarrollo jurídico avanza de acuerdo con los cambios que hemos venido señalando, es el relativo a la responsabilidad internacional, cuya aplicación en la actualidad no está solamente dirigida a los Estados sino también a las organizaciones internacionales y al individuo.

Como todos sabemos, el tema de la responsabilidad internacional, en lo que respecta a los Estados, se viene estudiando en el seno de la Comisión de Derecho Internacional de Naciones Unidas (CDI) desde 1949.39 Sin embargo, recién en 1996, se redactó un proyecto de artículos centrado en tres partes: "El origen de la responsabilidad"; "Contenido, formas y grados de la responsabilidad internacional"; y "Solución de controversias". Este proyecto se ha seguido perfeccionando, aunque aún no ha sido aprobado definitivamente. Una de las cuestiones más polémicas es la distinción entre crímenes y delitos internacionales. ${ }^{40}$ En el primer proyecto preparado por la Comisión de Derecho Internacional, se define al "crimen internacional", por ejemplo, como aquel que resulta de la violación por un Estado de una obligación internacional que es esencial para salvaguardar los intereses fundamentales de la comunidad internacional.

39 En 1949, la Comisión de Derecho Internacional de Naciones Unidas en su primer periodo de sesiones, seleccionó el tema de la "Responsabilidad Internacional de los Estados", como tema esencial para avanzar hacia una codificación. En 1976, como resultado de los trabajos de la CDI en esta temática, incorporó la distinción entre crímenes y delitos internacionales en el artículo 19 del Proyecto sobre Responsabilidad Internacional de los Estados. En 1980, la primera parte del Proyecto sobre Origen de la Responsabilidad Internacional fue aprobado. En 1996, se aprueba también en primera lectura, la segunda parte (contenido, formas y grados de responsabilidad internacional) y la tercera parte (solución de controversias y modalidades para hacer efectiva la responsabilidad.) En 2001, el proyecto fue aprobado en una segunda lectura, con un nuevo título: "Responsabilidad del Estado por hechos internacionalmente ilícitos". En este proyecto desapareció la distinción entre crímenes y delitos internacionales, pero en su artículo 26 se establece "Que ninguna disposición... excluirá la ilicitud de cualquier hecho del Estado que no esté de conformidad con una obligación que emana de una norma imperativa de derecho internacional". Véase los informes anuales de la Comisión de Derecho Internacional de las Naciones Unidas, donde aparece una relación pormenorizada de todos sus trabajos, incluyendo esta materia.

40 Véase artículo 19 del proyecto sobre responsabilidad internacional, CDI, Naciones Unidas. 
Otro aspecto interesante es que se considera la posibilidad de una actio popularis que permite, ante la violación de una norma de jus cogens, es decir que afecta a la comunidad internacional en su conjunto, la reacción no solamente del Estado afectado directamente sino también de terceros Estados.

Además de estos avances en materia de responsabilidad internacional de los Estados, cuya codificación aún no está consolidada, aparece otro desarrollo importante, en consonancia con la evolución de los principios que señalábamos más arriba, y es el relativo a la responsabilidad penal del individuo. Cabe anotar que el proyecto de Código de Crímenes contra la Paz y la Seguridad de la Humanidad, aprobado por la CDI en 1996, establece que "El hecho de que el presente Código prevea la responsabilidad de las personas por crímenes contra la paz y la seguridad de la humanidad no prejuzga ninguna cuestión de la responsabilidad internacional de los Estados en virtud del derecho internacional". ${ }^{41}$ El avance está dado en una clara separación entre la responsabilidad del Estado y la del individuo. Esta evolución conceptual ha sido importante para la creación de tribunales penales internacionales para el juzgamiento y castigo de individuos culpables de crímenes internacionales.

Por su parte, desde el 2000, el tema de la responsabilidad internacional de las organizaciones internacionales se viene estudiando en el marco de la Comisión de Derecho Internacional de Naciones Unidas. La responsabilidad de las organizaciones internacionales, consideradas como sujetos de derecho internacional con personalidad jurídica propia, diferente a las de sus Estados miembros u otras entidades, está concebida de manera tal que podrá ser exigida tanto por hechos internacionalmente ilícitos realizados a través de actos de los órganos de dicha organización internacional (responsability), como también por las consecuencias perjudiciales o daños causados a través de actos no prohibidos por el derecho internacional (liability). ${ }^{42}$

En resumen, son muchos y muy importantes los cambios que se vienen operando a nivel del derecho internacional en razón de las nuevas realidades en las que vivimos; el reto se encuentra ahora en avanzar en el

41 Véase artículo 4o. del Proyecto de Código de Crímenes contra la Paz y la Seguridad de la Humanidad, aprobado por la CDI/NU en 1996.

42 Sobre los últimos avances en el tema de la responsabilidad internacional de las organizaciones internacionales, véase el Informe de la Comisión de Derecho Internacional presentado en su 55 Periodo de Sesiones en 2003. 
camino del desarrollo y la codificación del derecho internacional, sin perder de vista los valores fundamentales básicos y la coherencia que necesita un orden jurídico que continúa estando inscrito en una sociedad internacional con un carácter descentralizado a pesar de la globalización, muy lejana aún de un gobierno mundial.

\section{EL DERECHO INTERNACIONAL EN EL MARCO DEL SISTEMA INTERAMERICANO}

En el sistema interamericano de inicios del siglo XXI, constatamos que los cambios ocurridos a nivel mundial han tenido también un gran impacto en nuestro ámbito regional, donde se aprecia el afianzamiento progresivo del Estado de derecho y las economías de mercado, con la presencia de gobiernos democráticos en prácticamente todo el hemisferio, que han reemplazado el autoritarismo y las divisiones ideológicas que primaban en el pasado, por la solidaridad y la cooperación. Al mismo tiempo, en un fenómeno complejo y simultáneo, todos los países se ven enfrentados a una serie de amenazas comunes como el narcotráfico, el terrorismo, el tráfico ilícito de armas, la corrupción, ${ }^{43}$ la inequidad y pobreza extrema, entre otros, que se ciernen sobre nuestros países amenazando los logros alcanzados.

Es un hecho objetivo que los condicionantes externos ejercen una decisiva y creciente influencia sobre los gobiernos y sobre la vida cotidiana de las personas, y que, a su vez, los asuntos internos tienen implicaciones a nivel internacional. Existe también la percepción de que un Estado no puede, aisladamente, hacer frente a sus necesidades, garantizar su desarrollo y la seguridad de sus nacionales.

En este contexto, apreciamos que las organizaciones y organismos internacionales, así como otros procesos multilaterales, como la Cumbre de las Américas y las reuniones hemisféricas de ministros (de Justicia, Trabajo, Comercio, Mujer, etcétera), tienen cada vez un rol más significativo y una mayor influencia que en el pasado. Tal es el caso de la Organización de Estados Americanos (OEA), principal foro político del hemisferio, donde los gobiernos y otras entidades — como las organiza-

43 En lo relativo al tema de la lucha contra la corrupción en la OEA, véase Lagos, Enrique, "La Organización de los Estados Americanos y la Lucha contra la Corrupción", 25o. Curso de derecho internacional, Secretaría General, Comité Jurídico Interamericano, OEA, 1998, pp. 231-254. 
ciones de la sociedad civil — se reúnen para considerar, negociar y adoptar diversas medidas que les permitan de manera conjunta, abordar temas y problemas de interés común de los Estados miembros, trabajando en torno al consenso y la coordinación de políticas, las responsabilidades compartidas, los mecanismos de seguimiento y, en determinadas circunstancias, adoptando medidas de acción colectiva. Esfuerzos conjuntos que son en la actualidad los ejes que guían el multilateralismo en el sistema interamericano, en sintonía con la actual realidad globalizada.

Con relación a la actual agenda hemisférica puede apreciarse una gran diversidad temática, reflejada por ejemplo en los acuerdos adoptados en la última Cumbre de las Américas, de Quebec City en 2001, y en la última Asamblea General de la OEA, realizada en Santiago de Chile en 2003. Agenda en donde se aprecia la convergencia de valores y principios comunes, encontrándose en la base del consenso la aceptación de las formas democráticas de gobierno, el respeto irrestricto a los derechos humanos, y la necesidad de fomentar el libre comercio y las economías de mercado, coadyuvando a producir un conjunto de objetivos interconectados y comunes.

Por su parte, el desarrollo y la codificación del derecho internacional en la región, ha evolucionado de acuerdo a los hechos antes mencionados, abarcando un amplio espectro vinculado a la consolidación y defensa de la democracia, la protección de los derechos humanos, la promoción de la justicia social, el crecimiento económico con equidad a favor de un desarrollo integral y sostenible, así como la seguridad colectiva en una concepción multidimensional que trasciende lo exclusivamente militar.

En todos estos temas, de acuerdo con las exigencias de los países, se constata nuevas modalidades de elaboración de las normas, recomendaciones y directrices a través de un proceso más informal y dinámico (soft law). En este sentido, la $\mathrm{OEA}^{44}$ ha constituido un foro privilegiado para promover la cooperación entre los Estados miembros a través de prácticas comunes, estándares de conducta y mecanismos de seguimiento de los acuerdos adoptados.

Gran parte de estos temas son claramente trasnacionales y aún los problemas tradicionalmente internos, como el fortalecimiento de las ins-

44 Véase Lagos, Enrique y Rudy, T., "The Organization of American States (OAS)”, International Encyclopedia of Laws, The Hague, Kluwer Law International, 2001. 
tituciones, la pobreza, la seguridad ciudadana y la corrupción, están hoy en día inscritos en la agenda multilateral. Aunque los gobiernos nacionales tengan la primera responsabilidad para enfrentar estos desafíos, existe la convicción de que en un mundo interdependiente, sólo a través de la cooperación y la complementación de acciones multilaterales con las políticas nacionales, se podrá avanzar en resolver los problemas que afectan, en mayor o menor medida, a todos los países del continente.

Precisamente, en lo que respecta a la democracia representativa, yendo incluso más allá de los avances que existen a nivel universal, encontramos que ésta constituye en la actualidad la única modalidad política legítima que se acepta en el ámbito multilateral interamericano (OEA, Cumbre de las Américas, Mercosur, Comunidad Andina, etcétera). La exigencia democrática para el sistema político, en el caso de la OEA, se encuentra en la propia carta constitutiva que exige el cumplimiento efectivo de la misma, contemplándose en una doble vertiente: de un lado, como supuesto o condición (para el logro de valores superiores como la paz, la estabilidad y el desarrollo; los derechos humanos, la justicia social, etcétera) y por otro, como propósito esencial de la propia organización; lo cual ha llevado a un nuevo alcance de las obligaciones internacionales de la OEA en relación a la promoción y defensa de la democracia.

Para reafirmar estos principios e implementar un proceso efectivo de defensa de la democracia y el Estado de derecho, en 1991, se aprobó la Resolución 1080; en 1992, el Protocolo de Washington (artículo 9), ${ }^{45}$ y en 2001, se aprueba la Carta Democrática Interamericana, que contiene principios, normas y mecanismos de acción colectiva, incluyendo sanciones diplomáticas, para los casos de alteración o ruptura de la institucionalidad democrática, con la finalidad de promover, preservar y defender

45 En 1991, la XXI Asamblea General de la OEA aprueba "El compromiso de Santiago con la democracia y la renovación del sistema interamericano"; la Resolución 1080 sobre Democracia Representativa que contiene el primer mecanismo de acción colectiva de la OEA frente a una interrupción ilegal del proceso democrático en un Estado miembro; y la Resolución 1124 que crea la Unidad para la Promoción de la Democracia en la OEA. En 1992, el Protocolo de Washington incorpora a la Carta de la OEA en el artículo 9o.: " la suspensión del ejercicio de participación en los órganos y entidades de la OEA a un Estado miembro cuyo gobierno democráticamente constituido haya sido derrocado por la fuerza". 
la democracia en una perspectiva de respeto de los derechos humanos, reconociendo que la democracia es un derecho de los pueblos. ${ }^{46}$

Cabe destacar que en el caso de las medidas que conllevan una acción colectiva concertada para lograr el cumplimiento de propósitos y principios comunes, como los contenidos en la Carta de la OEA e instrumentos interamericanos, éstas han superado la antigua barrera de la no-intervención. Así, por ejemplo, mediando ciertos procedimientos, la acción colectiva puede utilizarse por parte de la OEA para responder rápidamente en defensa de la democracia representativa en sus Estados miembros en casos de crisis, no considerándose que dicha acción constituya una "intervención en los asuntos internos de un Estado", sino un mecanismo aceptado y que resulta más beneficioso y menos costoso que las acciones unilaterales.

Otra área fundamental, concordante con los desarrollos a nivel internacional, es la relativa a la protección y promoción de los derechos humanos. El sistema interamericano de derechos humanos pasó de valores proclamados en declaraciones y resoluciones al cumplimiento obligatorio contenido en la Convención Americana sobre Derechos Humanos y otros tratados, donde además de proclamarse los derechos de la persona humana a través de distintos mecanismos e instituciones ${ }^{47}$, constituye una garantía colectiva poniendo a disposición del individuo diversos mecanismos legales para la defensa de sus derechos, dignidad y libertad, cuando la jurisdicción estatal se muestra indiferente o carece de eficacia para resolver los abusos y violaciones a los derechos fundamentales de las personas.

También en concordancia con la globalización económica, dentro de un esquema de regionalismo abierto, los gobiernos del continente vienen negociando con el apoyo de entidades interamericanas (OEA, Banco Interamericano de Desarrollo (BID), Comisión Económica para América Latina, CEPAL, etcétera) la posible creación de un Área de Libre Comercio de las Américas (ALCA) para el 2005. En este sentido, las normas jurídicas, tanto a nivel del derecho internacional publico como en

46 Véase Lagos, Enrique y Rudy, T., "The Third Summit of the Americas and the Thirty First Session of the OAS General Assembly", American Journal of International Law, vol. 96, núm. 1, 2002.

47 Véase en página Web de la OEA (www.oas.org) todos los mecanismos y procedimientos para la defensa de los derechos del individuo llevados adelante por la Comisión Interamericana de Derechos Humanos y la Corte Interamericana de Derechos Humanos. 
del derecho internacional privado, vienen experimentando importantes desarrollos.

En este contexto, la OEA es el único foro de las Américas que posee una representatividad que congrega a todos los países del hemisferio, y que cuenta con una basta experiencia y tradición en materia de codificación y desarrollo jurídico. Un ejemplo relevante y demostrativo de lo expuesto se encuentra en el proceso comenzado a mediados de los años setenta, con la Conferencia Interamericana sobre Derecho Internacional Privado (CIDIP).

Este órgano de la OEA, tradicionalmente ha focalizado su trabajo en la codificación de las normas de derecho internacional privado y, como resultado de sus labores, ha adoptado más de veinte instrumentos jurídicos, la mayoría de los cuales se encuentran actualmente en vigor.

En la última década, sin embargo, la CIDIP ha venido reduciendo el tratamiento de la codificación de normas de derecho internacional privado, ampliando sus actividades hacia asuntos más ligados a la armonización de legislaciones nacionales de derecho sustantivo o de carácter regulatorio vinculados con el derecho comercial y financiero, principalmente.

Dicha tendencia, es un claro reflejo de las actuales realidades que conlleva la globalización, y el impacto que este fenómeno representa en el plano jurídico, tanto nacional como internacional. Asimismo, estas circunstancias conducen a los Estados a interesarse en uniformar o al menos armonizar sus ordenamientos legales para avanzar en áreas relacionadas con diversos sectores de sus economías y con políticas comerciales ligadas a los esquemas de integración económica u otras regulaciones legales que les permitan desenvolverse mejor dentro del mundo actual.

En lo que respecta a la seguridad hemisférica, después de los ataques terroristas de septiembre de 2001, una nueva dimensión de políticas de cooperación se ha venido gestando a nivel interamericano. Por un lado, están las iniciativas para llevar adelante la lucha contra el terrorismo, dentro de un renovado marco jurídico concordante con el de Naciones Unidas, la Convención Interamericana contra el Terrorismo, ${ }^{48}$ así como a

48 La Convención Interamericana contra el Terrorismo fue aprobada en la Asamblea General de la OEA en junio de 2002 realizada en Bahamas. Actualmente se encuentra en vigor. Véase Lagos, Enrique y Rudy, T., "Preventing, Punishing and Eliminating Terrorism in The Western Hemisphere: A post 9/11 Inter-American Treaty", Fordham International Law Journal, Nueva York, Fordham University School of Law, vol. 26, junio de 2003, núm. 6, pp. 1619-1648. 
un conjunto de estrategias de cooperación diseñadas para combatir este flagelo, particularmente a través de las acciones del Comité Interamericano contra el Terrorismo (CICTE) ${ }^{49}$ Por el otro, después de un intenso proceso de negociación, se acaba de aprobar en México una Declaración sobre Seguridad en las Américas, que recoge no sólo los aspectos tradicionalmente considerados como asuntos de seguridad, vale decir, los vinculados a la defensa común de carácter militar frente a agresiones externas, sino que también se considera ahora una diversidad y pluralidad de intereses de seguridad contenidos en una conceptualización multidimensional frente a las nuevas amenazas, preocupaciones y otros desafíos como son la pobreza y la exclusión social, la defensa de la democracia, la ayuda frente a los desastres naturales y una serie de otros factores que es necesario tener en cuenta para la seguridad, protección y bienestar de la persona humana en todas sus dimensiones y en forma integral. ${ }^{50}$

\section{CONCLUSIONES}

Las reflexiones antes vertidas dejan claro que el vasto y dinámico mundo actual está marcado con el signo de la globalización y la interdependencia, que marchan de la mano con la revolución informática y de las comunicaciones. Vivimos en una sociedad uni-multipolar compleja, en la que se entrecruzan los más diversos actores que ejercen el poder de diversas formas y en los distintos planos del escenario internacional. Así, el Estado que sigue siendo el sujeto por excelencia del derecho internacional, hoy en día comparte roles con otros sujetos, como las organizaciones internacionales, diversas entidades de la sociedad civil y empresas multinacionales.

En este contexto, los Estados y todos los actores a nivel supranacional, cada vez más, convergen en determinados valores comunes, fundados en identidad de propósitos, metas y medios, de forma tal, que también podríamos proyectar a un futuro cercano la globalización de una

49 El Comité Interamericano contra el Terrorismo (CICTE) como entidad de la OEA, fue creado a través del "Compromiso de Mar del Plata", durante la II Conferencia Especializada sobre Terrorismo de la OEA, realizada en Mar del Plata, Argentina, en 1998.

50 Véase en página Web (www.OAS.org), la Declaración de Seguridad en las Américas, aprobada en la Conferencia Especial de Seguridad de la OEA realizada en México el 27 y 28 de octubre de 2003. 
ética común, que tenga como base y norte el respeto y la protección de la vida y la dignidad humana de las generaciones presentes y futuras.

El multilateralismo, que se hace efectivo a través de las organizaciones internacionales, organismos, entidades interestatales y otros variadas modalidades de coordinación supranacional, hace posible superar, en un mundo interdependiente, las deficiencias de un relacionamiento sólo bilateral, integrando a numerosos actores en las negociaciones y búsqueda de soluciones a los problemas comunes, trascendiendo los intereses puramente nacionales por valores comunes de toda la humanidad. De este modo, las acciones que se acuerdan están fundamentalmente basadas en la solidaridad y en la cooperación internacional.

El desarrollo y la codificación del derecho internacional, al compás de las nuevas exigencias del mundo actual, está experimentando notables cambios; así por ejemplo, ha incorporado una notable ampliación en su temática contemplando prácticamente todos los temas de interés nacional; existen nuevas modalidades en los procesos de elaboración de las normas; se están realizando avances significativos en el tema de la responsabilidad internacional, abarcando en el estudio de esta temática no sólo a los Estados sino también a las organizaciones internacionales y al individuo; todo esto da como resultado una compleja gama de innovaciones tanto en los aspectos sustantivos, institucionales como en los de procedimiento.

En el ámbito interamericano, se está logrando una convergencia creciente, marcada por una comunidad de valores e ideales centrados en el respeto a la democracia, a los derechos humanos y a la necesidad de construir sociedades más justas y equitativas, donde prevalezca la paz, el bienestar y la seguridad para todos. Los desafíos a estas aspiraciones son enormes, pero también las oportunidades, y en esta línea el derecho internacional interamericano presenta desarrollos muy importantes que son reafirmados por la firme creencia de que el cumplimiento de los compromisos internacionales a través de la cooperación y la solidaridad son las bases fundamentales para construir una comunidad de democracias en las Américas. 